\title{
First-pass cardiac PET: Potentiality and limitations
}

\author{
Luca Camoni, ${ }^{\mathrm{a}}$ and Elisabetta Cerudelli ${ }^{\mathrm{a}}$ \\ a Nuclear Medicine, University of Brescia, Brescia, Italy \\ Received Nov 30, 2020; accepted Nov 30, 2020 \\ doi: $10.1007 / \mathrm{s} 12350-020-02476-8$
}

\section{See related article, pp. 1003-1017}

Myocardial infarction (MI) and the consequent heart failure are the leading cause of death in the world population. ${ }^{1}$ Increasing importance are assuming the evidence and the extent of regional and global cardiac disfunction in coronary artery disease, not only as criteria in diagnosis of heart failure, but also as predictors of the clinical outcome. ${ }^{2}$ Coronary heart disease and MI are typically associated with increase of Left Ventricular (LV) and Right Ventricular (RV) volumes and the reduction of LV Ejection Fraction (EF). As indicators of impaired systolic function, several studies have documented the power of the volumetric parameters in prediction of a poor long-term prognosis. An accurate assessment of $\mathrm{LV}$ function in patients with prior MI assumed a fundamental role in the management and prevention of cardiovascular disease. ${ }^{3}$

Several studies have documented the feasibility of Gated 18F-fluorodeoxyglucose (FDG) positron emission tomography (PET) for assessment of left ventricular volumes and ejection fraction, in comparison with magnetic resonance imaging (MRI). ${ }^{4-8}$

In this issue of The Journal of Nuclear Cardiology, Rasul et al. ${ }^{9}$ report on the quantification of LV and RV volumes and EF using a dual-tracer PET scan integrated with MRI. In particular, they analysed the functional parameters derived from different phases of dynamic FDG and 13N-NH3 (NH3) PET scans and compared results to MRI, which was identified as the reference standard. The three phases were: first-pass, early and late

\footnotetext{
Reprint requests: Luca Camoni, Nuclear Medicine, University of Brescia, Brescia, Italy; camoni.luca@gmail.com J Nucl Cardiol 2022;29:1018-20. $1071-3581 / \$ 34.00$

Copyright (C) 2021 American Society of Nuclear Cardiology.
}

imaging. For this study, they retrospectively analysed 22 patients with coronary disease who underwent a dual tracer NH3 and FDG protocol to assess myocardial perfusion and viability.

The authors reported on the overall diagnostic power of PET imaging to define the parameters of LV function by both with FDG and NH3. Using the two tracers, authors found a strong correlation in two of the three sets of images: when the early acquisition time was complied with the guidelines ${ }^{10}$ and when performed in late imaging.

They confirmed the feasibility of using Gated FDG PET to derive LV parameters and its strong correlation with MRI. Besides, they reached the same results also using NH3, where relatively few works have been published. $^{11,12}$

Their data emphasize what is known in the Gated PET imaging: the suitability of the FDG and the NH3 to derive $\mathrm{LV}$ functional parameters, in comparison to a gold-standard technique.

The set of images obtained by authors from the FDG-first-pass deserves a mention. They have pushed the traditional idea of the standardized PET whole-body acquisition protocol, trying to evaluate the LV functional parameters using the first-pass FDG, in order to apply the technique to identify chemotherapy-induced cardiotoxicity in oncological patients.

Cardiovascular diseases demonstrated an increase of the prevalence among oncological patients. New therapies have resulted in significantly improved cancer survival rates, but on the other side more aggressive antineoplastic therapies, including biological and immunologic ones, have been developed. Some of these therapies, like anthracyclines or trastuzumab, are associated with cardiotoxicity that has emerged as a leading cause of morbidity in this kind of patients. Among the toxic effects the most frequent and serious is heart failure with ventricular systolic disfunction. ${ }^{13}$ In order to evaluate chemotherapy-induced cardiotoxicity, many types of examinations have been performed such as 
echocardiography, myocardial perfusion scintigraphy, multiple gated acquisition (MUGA) scintigraphy or cardiac MRI. In this context FDG-PET/CT or PET/MRI, widely used in the evaluation of oncological patients, has been demonstrated new utility. The increased uptake of the LV or the presence of RV wall uptake showed an association with chemotherapy-induced cardiotoxicity. ${ }^{14}$ Other signs of the presence of cardiovascular adverse effects are the increase of the LV end diastolic and systolic volume, the reduction of the EF and the LV hypertrophy. Different approaches are validated in the literature for the evaluation of parameters of LV function by PET. ${ }^{6,8}$ Following the experience first-pass radionuclide angiography using $99 \mathrm{mTc}$-labeled agents, first-pass PET methods were developed to evaluate RV and LV function. In this way, the first-pass of the tracer through cardiac cavities produces a series of vascular high-count images of the blood pool, that indirectly represents cardiac function, enabling ECG-gated blood pool imaging.

Any radionuclide can be used for first-pass imaging, but obviously, in the oncology field, a top-notch place can be assumed by FDG, the tracer most widespread for oncologic purposes.

Starting from this idea, the authors, thanks to the hybrid scanner PET/MRI, have tried to evaluate if the first-pass analysis can be translated in the PET/CT systems, to obtain RV and LV functional parameters.

This technique was developed in a clinical setting and the method seems to be effective for PET/CT, but limitations must be taken into account. Few articles were published on first-pass cardiac study using PET for assessing left ventricular function, mainly by $\mathrm{H}_{2} \mathrm{O}^{15}{ }^{15-18}$ In these studies, a strong correlation between first-pass PET and CMR functional data was found $(r>0.8)$, using automatic or semi-automatic software for cardiac wall detection. No studies directly compared manual vs. automatic contour selection in PET imaging. Therefore, the manual selection could be one of the main limitations in this study and it could justify the underestimation of overall EF. In the study limitations, it is underlined that the manual selection could be one the reasons of the poor intra-individual correlation between first-pass PET and CMR but this should be further emphasized.

The filling and emptying phases of the heart require an accurate wall selection. The delineation of atria and ventricles, in the diastolic and systolic phases, must ensure the correct inclusion of each chambers and exclude any counts interference between themselves. Applying a count-based calculations and automated delineation of myocardium helps to track the walls movement during the phases. The opposite is a manual delineation in a single frame in diastole and at end systole without the help of phase analysis for identifying the valvular plane.

Moreover, not only nuclear imaging but also cardiac MRI has methodological limitations in the assessment of myocardial volumes. First the inclusion or exclusion of trabecular myocardium in the count, but also the definition of the final basal LV slice, gating artifacts, and its need for consistent multiple breath-holds for diagnostic images. $^{19}$

Whether a region contouring is erroneous, it completely fails the evaluation of the function parameters in some cases, both in PET or MRI, moreover in the firstpass or blood pool, techniques based on counts interpretation.

Others limitations of this study, correctly enlightened by the authors are: the small sample size, the high prevalence of diabetic patients, different patient preparation and different protocols in the FDG- PET, the high administered activity for NH3. Count saturation is a well-known problem in PET imaging and the maximal count rate should be below the saturation threshold according to the noise equivalent count rate curves of each specific system.

Nonetheless, between the limitations, the procedure is also time expensive when adopted in FDG-PET due to the need of double patient positioning and centering for early (first-pass) and late acquisition. Moreover, whether the technique is extended to the PET/CT, this approach has another disadvantage due to the double CT scanning for emission PET data correction for random coincidences, scatter and dead time, applying the CT-based attenuation correction for both first-pass and wholebody. This introduces an additional effective dose due to cardiac CT. ${ }^{15,20}$

Future researches are needed to address the limitations in first-pass cardiac PET imaging, measurements still require more appropriate standardization and an adequate software development. However, the results of the study are interesting, opening a new perspective for the evaluation of ventricular function in FDG PET acquired for oncological purposes.

\section{References}

1. Global, regional, and national age-sex-specific mortality for 282 causes of death in 195 countries and territories, 1980-2017: A systematic analysis for the Global Burden of Disease Study 2017. Lancet 2018;392:1736-88.

2. Hammermeister KE, DeRouen TA, Dodge HT. Variables predictive of survival in patients with coronary disease. Selection by univariate and multivariate analyses from the clinical, electrocardiographic, exercise, arteriographic, and quantitative angiographic evaluations. Circulation 1979;59:421-30.

3. White HD, Norris RM, Brown MA, Brandt PW, Whitlock RM, Wild CJ. Left ventricular end-systolic volume as the major 
determinant of survival after recovery from myocardial infarction. Circulation 1987;76:44-51.

4. Higuchi T, Nekolla SG, Jankaukas A, Weber AW, Huisman MC, Reder S, et al. Characterization of normal and infarcted rat myocardium using a combination of small-animal PET and clinical MRI. J Nucl Med 2007;48:288-94.

5. Khorsand A, Graf S, Frank H, Kletter K, Sochor H, Maurer G, et al. Model-based analysis of electrocardiography-gated cardiac (18)F-FDG PET images to assess left ventricular geometry and contractile function. J Nucl Med 2003;44:1741-6.

6. Li Y, Wang L, Zhao SH, He ZX, Wang DY, Guo F, et al. Gated F18 FDG PET for assessment of left ventricular volumes and ejection fraction using QGS and 4D-MSPECT in patients with heart failure: A comparison with cardiac MRI. PLoS ONE 2014;9:e80227.

7. Schaefer WM, Lipke CS, Nowak B, Kaiser HJ, Reinartz $P$, Buecker A, et al. Validation of QGS and 4D-MSPECT for quantification of left ventricular volumes and ejection fraction from gated 18F-FDG PET: Comparison with cardiac MRI. J Nucl Med 2004;45:74-9.

8. Slart RH, Bax JJ, de Jong RM, de Boer J, Lamb HJ, Mook PH, et al. Comparison of gated PET with MRI for evaluation of left ventricular function in patients with coronary artery disease. J Nucl Med 2004;45:176-82.

9. Rasul S, Beitzke D, Wollenweber T, Rausch I, Lassen ML, Stelzmüller ME, et al. Assessment of left and right ventricular functional parameters using dynamic dual-tracer [13N]NH3 and [18F]FDG PET/MRI. J Nucl Cardiol 2020.

10. Dilsizian V, Bacharach SL, Beanlands RS, Bergmann SR, Delbeke $\mathrm{D}$, Dorbala $\mathrm{S}$, et al. ASNC imaging guidelines/SNMMI procedure standard for positron emission tomography (PET) nuclear cardiology procedures. J Nucl Cardiol 2016;23:1187-226.

11. Nazir MS, Gould SM, Milidonis X, Reyes E, Ismail TF, Neji R, et al. Simultaneous (13)N-Ammonia and gadolinium first-pass myocardial perfusion with quantitative hybrid PET-MR imaging: A phantom and clinical feasibility study. Eur J Hybrid Imaging 2019;3:15
12. Okazawa H, Takahashi M, Hata T, Sugimoto K, Kishibe Y, Tsuji T. Quantitative evaluation of myocardial blood flow and ejection fraction with a single dose of (13)NH(3) and Gated PET. J Nucl Med 2002;43:999-1005.

13. Broder H, Gottlieb RA, Lepor NE. Chemotherapy and cardiotoxicity. Rev Cardiovasc Med 2008;9:75-83.

14. Kim J, Cho SG, Kang SR, Yoo SW, Kwon SY, Min JJ, et al. Association between FDG uptake in the right ventricular myocardium and cancer therapy-induced cardiotoxicity. J Nucl Cardiol 2019.

15. Ben Bouallègue F, Maïmoun L, Kucharczak F, Le Fur P, Vauchot F, Hay B, et al. Left ventricle function assessment using gated first-pass (18)F-FDG PET: Validation against equilibrium radionuclide angiography. J Nucl Cardiol 2019.

16. Ben Bouallègue F, Mariano-Goulart D, Agostini D, Manrique A. Feasibility of biventricular volume and function assessment using first-pass gated (15)O-water PET. EJNMMI Res 2018;8:92.

17. Driessen RS, van Timmeren JE, Stuijfzand WJ, Rijnierse MT, Danad I, Raijmakers PG, et al. Measurement of LV volumes and function using oxygen-15 water-gated PET and comparison with CMR imaging. JACC Cardiovasc Imaging 2016;9:1472-4.

18. Nordström J, Kero T, Harms HJ, Widström C, Flachskampf FA, Sörensen J, et al. Calculation of left ventricular volumes and ejection fraction from dynamic cardiac-gated (15)O-water PET/ CT: 5D-PET. EJNMMI Phys 2017;4:26.

19. Chuang ML, Gona P, Hautvast GL, Salton CJ, Blease SJ, Yeon $\mathrm{SB}$, et al. Correlation of trabeculae and papillary muscles with clinical and cardiac characteristics and impact on CMR measures of LV anatomy and function. JACC Cardiovasc Imaging 2012;5:1115-23.

20. Camoni L, Santos A, Attard M, Mada MO, Pietrzak AK, Rac S, et al. Best practice for the nuclear medicine technologist in CTbased attenuation correction and calcium score for nuclear cardiology. Eur J Hybrid Imaging 2020;4:11

Publisher's Note Springer Nature remains neutral with regard to jurisdictional claims in published maps and institutional affiliations. 